\title{
Orofacial Manifestations of Encephalotrigeminal Angiomatosis: A Case Report and Review of Literature
}

\author{
V. Bhavana, B. Balaji Babu, J. Shilpa Rao, M. Mounika Reddy \\ Department of Oral Medicine and Radiology, Kamineni Institute of Dental Sciences Narketpally, Nalgonda, Telangana, India
}

Email for correspondence: bhavana2966@gmail.com

\begin{abstract}
Sturge-Weber syndrome (SWS), also called as encephalotrigeminal angiomatosis, is a sporadically occurring neurocutaneous syndrome caused by persistence of transitory primordial arteriovenous connection of the fetal intracranial vasculature. It is characterized by vascular malformation with capillary venous angiomas that involve face, choroid of eye, and leptomeninges with resulting neurological and orbital manifestations. The diagnosis is usually considered in a child presenting with seizures and facial capillary malformation along the trigeminal nerve distribution. In this article, a case of SWS associated with oral manifestations and review on literature is presented.
\end{abstract}

Key words: Port-wine stain, sturge--weber syndrome, tram-track calcifications

\section{INTRODUCTION}

Sturge-Weber syndrome (SWS), or encephalotrigeminal angiomatosis, is a rare, congenital neurocutaneous syndrome characterized by unilateral facial cutaneous vascular malformation (nevus flammeus or port-wine stain [PWS]) in association with ipsilateral leptomeningeal angiomatosis. ${ }^{[1]}$ It was first described by Sturge in 1879 in a 6-year-old girl with facial nevus, and later, cerebral involvement was demonstrated AQ2 by Kalischer in $1897^{[2]}$ It is often associated with seizures and other neurologic complications, including mental retardation, contralateral hemiparesis, and glaucoma. ${ }^{[3]}$ Prevalence of the disease among the neurocutaneous syndromes, especially with vascular predominance, is $1 / 50,000$ live births and no racial bias. ${ }^{[4]}$ Both the genders are affected equally. Intraorally, angiomatosis can involve lips causing macrocheilia, resulting

\begin{tabular}{|l|l|}
\hline Quick Response Code & Article Info: \\
\hline doi: 10.5866/2017.9.10226 \\
\hline $\begin{array}{l}\text { Received: } 16-12-201 \\
\text { Revised: } 29-12-2018 \\
\text { Accepted: 06-01-2018 } \\
\text { Available Online: 15-02-2018, 2018 (www. } \\
\text { nacd.in)@ NAD, 2018 - All rights reserved }\end{array}$ \\
\hline
\end{tabular}

in hemihypertrophy of the buccal mucosa, palate, and the floor of the mouth. Vascular hyperplasia is most commonly seen involving gingival. ${ }^{[4]}$ Failure of its regression results in residual vascular tissue which forms angiomas of leptomeninges, face and ipsilateral eye. These blood vessels show abnormal blood flow pattern as vasomotor phenomenon, venous occlusion, thrombosis and "vascular steal phenomenon" resulting in ischaemia, gliosis, atrophy and calcification of underlying cortical tissue. Although the leptomeningeal angioma in SWS is typically a static lesion, it has been demonstrated by some to be of progressive nature. ${ }^{[5}$

\section{CASE REPORT}

A 23-year-old male patient was referred to the Department of Oral Medicine and Radiology with a chief complaint of swollen gums. On medical history, he had no history of seizures. The patient was observed to have a normal intelligence, a moderate build, and normal temperature, pulse, respiration, and blood pressure. On extraoral examination, a hemihypertrophy was observed on the right side of the face extending from hairline till lower border of mandible along the division of the trigeminal nerve with PWS which was present since birth [Figure 1]. Mouth and nasal septum were deviated toward the right side of the face. 
Glaucoma was seen involving the eye [Figure 2]. Intraoral examination revealed soft, non-tender vascular hyperplasia involving gingiva in right upper quadrant of the oral cavity, and a solitary well-defined ulcer of size $0.8 \quad 1 \mathrm{~cm}$ is seen on swelling in gingiva due to trauma which is tender on palpation [Figure 3]. Red pigmentation was observed over the upper and lower labial mucosa [Figure 4], buccal mucosa, palate, and ventral surface of the tongue on the right side [Figure 5]. Hypertrophy of the upper and lower lip is present [Figure 6]. On diascopy test, blanching was seen in the upper and lower lip and maxillary gingival on the right side [Figure 7].

Differential diagnosis of Klippel-TrenaunayWeber syndrome in which PWSs are seen in

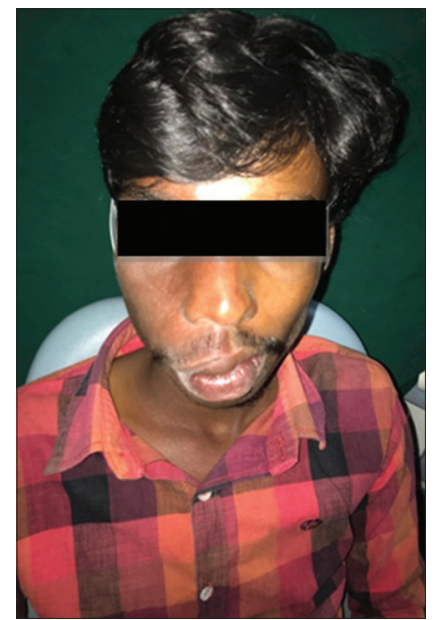

Figure 1: Clinical picture showing port-wine stain on the right side of face

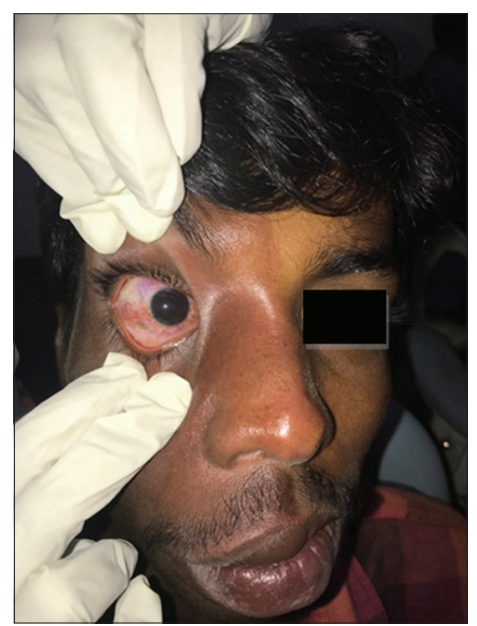

Figure 2: Clinical picture showing glaucoma involving the right eye extremities and face, hemihypertrophy of soft and bony tissues, and Beckwith-Wiedemann syndrome along with facial PWS, macroglossia, omphalocele, and visceral hyperplasia, other lesion syndrome, and coats disease were considered.

Investigations that were carried out in the present case included an orthopantomograph (OPG), lateral skull view, and complete hemogram. The OPG revealed hemifacial hypertrophy in the right side of face [Figure 8]. The complete hemogram showed normal hemoglobin levels with no other abnormality in the total or differential white blood cell counts. Bleeding and clotting time were within normal limits. Lateral skull view showed no abnormalities such as calcification, atrophy with dilatation of ventricle, and enlargement of sinuses. The presence of glaucoma was confirmed by ophthalmologist. Based on the clinical findings and radiological findings, diagnosis of SWS was given.

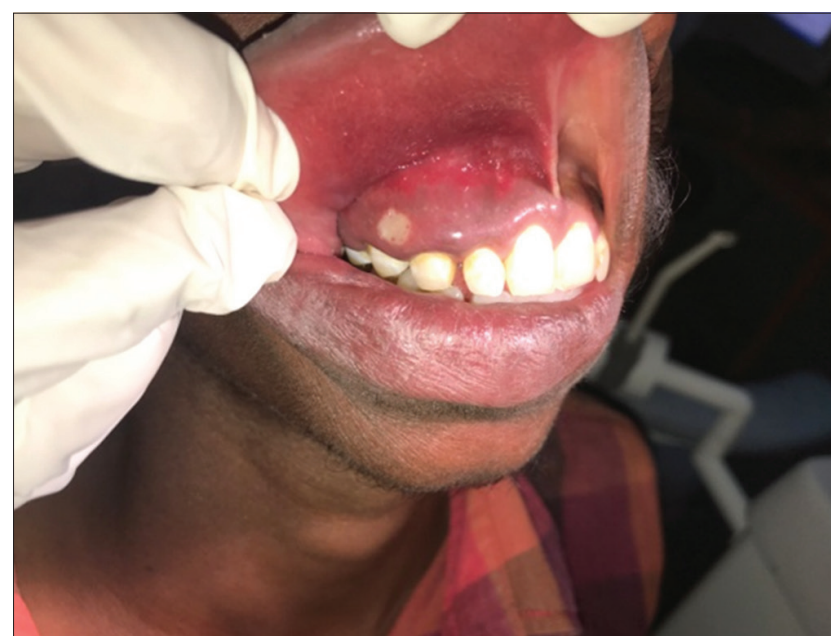

Figure 3: Intraoral examination showing vascular hyperplasia involving gingiva in right upper quadrant

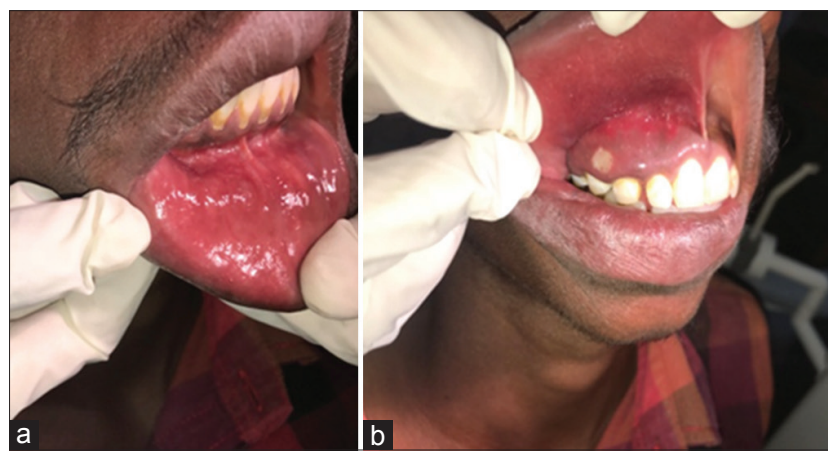

Figure 4: ( $a$ and b) Intraoral examination showing red pigmentation in the upper and lower labial mucosa 


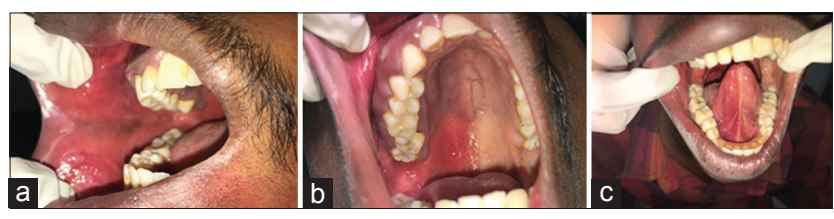

Figure 5: (a-c) Intraoral examination showing red pigmentation in the buccal mucosa, hard palate, and ventral surface of tongue on the right side

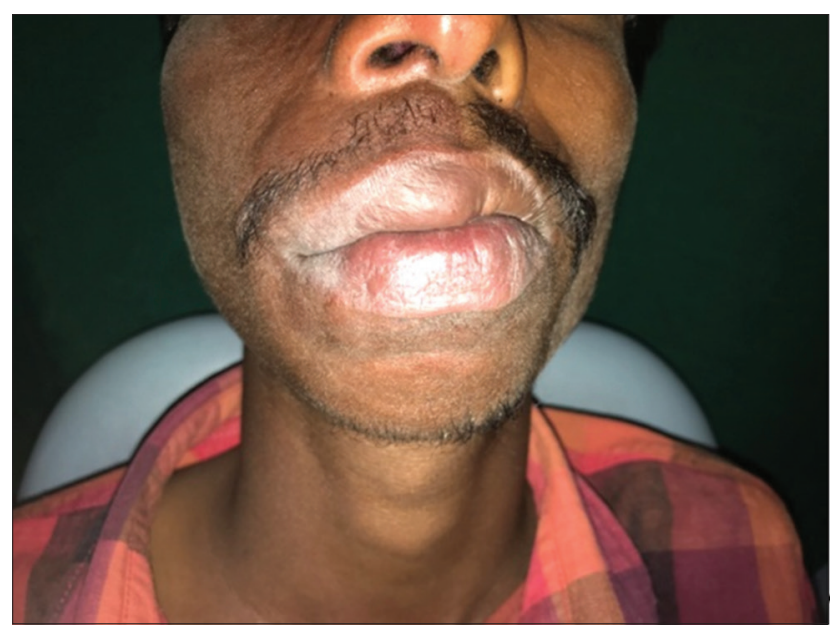

Figure 6: Hypertrophy of the upper and lower lip
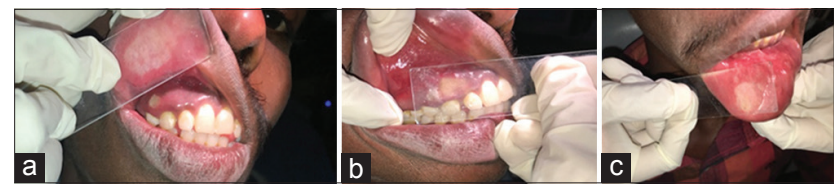

Figure 7: (a-c) Positive diascopy test

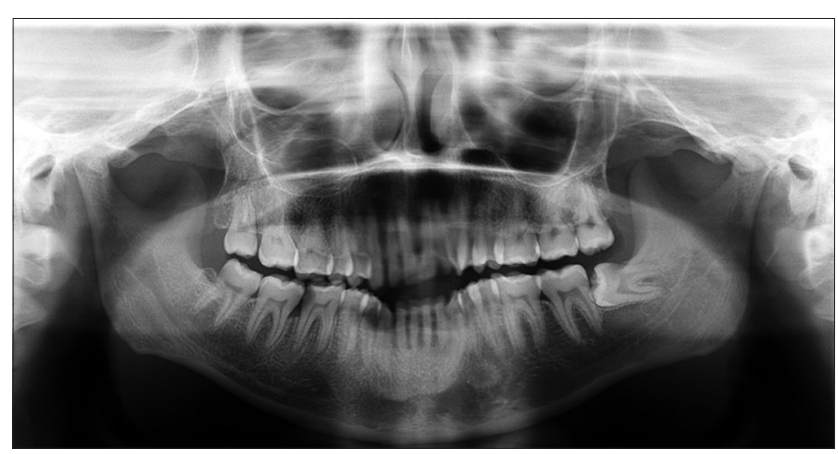

Figure 8: Orthopantomogram showing hemifacial hypertrophy is seen on right side

\section{DISCUSSION}

SWS is a typical neurocutaneous syndrome which is uncommon, non-familial, congenital condition of unknown etiology. It is often associated with capillary or cavernous hemangiomas affecting primarily but not limited to cutaneous distributions of the trigeminal nerve and rarely venous malformations involving leptomeninges that lead to progressive destruction of the adjunct cerebral cortex ${ }^{[6]}$ Most commonly involves one or more unit of trigeminal nerve unilaterally. ${ }^{[7]}$

It is believed that SWS results from a developmental defect in the $1^{\text {st }}$ month of gestation, represented by the persistence of a vascular plexus which develops in the $6^{\text {th }}$ week of intrauterine life, but normally undergoes regression during the $9^{\text {th }}$ week around the cephalic portion of the neural tube. The association between cerebral angiomatosis and facial nevus, characteristic of the syndrome, is attributed to the development of the skin of the face from the ectoderm that covers this vascular plexus. ${ }^{[3]}$

When both cranial nervous system and facial angiomas are present, SWS is referred to as complete and incomplete when only one area is affected without the other. It is classified into three types based on roach scale: Type I - both facial and Las - may have glaucoma, Type II - facial angioma alone (no central nervous system [CNS] involvement) - may have glaucoma, and Type III - isolated LAs - usually no glaucoma. ${ }^{[8]}$

In the present case, there was ocular involvement with glaucoma, and facial angioma was unilateral present since birth with vascular hyperplasia involving gingival in the affected quadrant (right maxillary) without any CNS manifestations leads to a diagnosis of SWS Type II according to Roach classification.

According to Inan, the port-wine nevus is localized on the face, especially over the right side, and is detected in $87-90 \%$ of the cases. The lesion extension over the middle line is observed in $50 \%$ of the patients and bilateral involvement can be detected in about $33 \%$ of the cases. ${ }^{[9]}$

The ipsilateral leptomeningeal angiomatosis also represents one of the main signs of the syndrome, may present with a progressive nature, and commonly leads to cerebral calcifications, epileptic convulsive crises, contralateral hypertrophy, hemiparesis (30\%), and mental retardation (50\%). ${ }^{[4}$ Facial lesions are the other characteristic feature of this syndrome and include rosy-purple nevus flammeus lesions that are sharply demarcated and usually flat; these occur on the ipsilateral side of the face in $90 \%$ of the patients, which may 
extend onto the neck, chest, and back. The facial features also may include hemihypertrophy of the involved side of the face. Ocular involvement may present in the form of choroidal angioma, glaucoma, hemianopsia, or buphthalmos (enlargement of the coating of the eye). Oral manifestations are present in approximately $38 \%$ of the cases, and they may involve hemangiomatous lesion in the lip, oral mucosa, gum, tongue, and palatine region. ${ }^{[10]}$

Neuroimaging studies include skull radiograph, angiography, CT scan, magnetic resonance imaging (MRI), MRI with gadolinium, and functional imaging with single-photon emission computed tomography (SPECT) or positron-emission tomography (PET). CT scan and skull radiograph may show the characteristic gyriform intracranial "tramline," or "tram-track" or "trolley-track," calcifications located in the cortex underlying the leptomeningeal vascular malformations, cerebral atrophy, and enlargement of the medullary and subependymal veins and the choroid plexus. ${ }^{[11]}$ Calcifications appear as areas of decreased signal intensity on spin density and T2-weighted MR images ${ }^{[12]}$ Cerebrospinal fluid shows elevated levels of proteins. Angiography shows lack of superficial cortical veins, non-filling dural sinuses, and abnormal torturous vessels. SPECT shows early hyperperfusion and late hypoperfusion. PET shows hypometabolism. Reduced background activity, polymorphic delta activity, and epileptiform features are seen in electroencephalogram. ${ }^{[13]}$

The primary aim of pharmacologic treatment is to minimize or optimally eliminate seizure activity. Anticonvulsants are most commonly used such as oxcarbazepine, levetiracetam, and topiramate. If seizures persist surgical procedures such as functional hemispherectomy, anatomic hemispherectomy, and hemispherectomy can be done. Opthalmologic symptom most commonly seen is glaucoma and can be effectively treated by topical medication latanoprost. Common surgical therapies include trabeculectomy, trabeculotomy, and goniotomy in infants and children, as well as valve drainage implants, non-penetrating sclerectomy, procedures in adults. ${ }^{[14]}$

Skin manifestations such as port-wine birthmark can be treated by lasers. Flashlamppumped PDL is the current method for lightening port-wine birthmarks, which targets the port-wine vasculature without affecting the surrounding epidermis or dermis. ${ }^{[15]}$
The dental rehabilitation in cognitive/ psychological function impairment patients is a complex process, requiring conservative management, and may demand the use of behavior management techniques due to mental retardation specialized education services, behavioral psychology intervention, and stimulant use in patients with cognitive deficits and attention problems have shown to be benefited. ${ }^{[16]}$ Conscious sedation can be used when required. Whenever oral surgery is planned in SWS patients, particular care must be paid to achieve hemostasis during and after the surgical procedure by splints, injection of anesthetic solutions with vasoconstrictors. In spite of strict oral hygiene measures, angiomatous gingival enlargement poses a threat to gingival health and may at some point require gingivectomy. $\mathrm{Nd}$ : $\mathrm{YAG}$ and $\mathrm{CO}_{2}$ lasers can be used for gingivectomies in SWS patients with good safety. ${ }^{[3]}$

\section{REFERENCES}

1. Godge P, Sharma S, Yadav M, Patil P, Kulkarni S. Sturge weber syndrome: A case report. Rev Odonto Cienc 2011;26:366-9.

2. Saroj G. Sturge weber syndrome with secondary glaucoma. J Clin Opthalmol Optom 2017;2(1):102.

3. Sharma N, Passi S, Mehta A. Sturge-weber syndrome: Report of a case and literature review. J Pediatr Dent 2014;2:65-9.

4. Neto FX, Junior MA, Ximenes LS, Jacob CCS, Junior AG, Palheta CP, et al. Clinical features of sturge-weber syndrome. Arq Int Otorrinolaringol 2008;12:565-70.

5. Wahab A. Sturge weber syndrome: A review. Bombay Hosp J 2008;50:641-3.

6. Sturge WA. A case of partial epilepsy, apparently due to a lesion of one of the Vaso motor centres of the brain. Trans Clin Soc Lond 1879;12:1627.

7. Bhansali RS, Yeltiwar RK, Agarwal AA. Periodontal management of gingival enlargement associated with sturgeweber syndrome. J Periodontol 2008;79:54955.

8. Roach ES. Neurocutaneous syndromes. Pediatr Clin North Am 1992;39:591-620.

9. Inan CJ. Sturge-weber syndrome: Report of an unusual cutaneous distribution. Brain Dev 1999;21:68-70.

10. Elavarasu S, Kumaran ST, Kumar PK. Periodontal management of gingival enlargement associated with sturge weber syndrome. J Ind Soc Periodontol 2013;17:235-8.

11. Akpinar E. The tram-track sign: Cortical calcifications. Radiology 2004;231:515-6.

12. Wilms G, Van Wijck E, Demaerel P. Gyriform calcifications in tuberous sclerosis simulating the appearance of sturgeweber disease. Am J Neuroradiol 1992;13:295-7. 
13. Kaur M. Orofacial manifestation of sturge-weber syndrome: A case report with review. J Orofacial Sci 2012;4:70-4.

14. Bachur CD, Comi AM. Sturge-weber syndrome. Curr Treat Options Neurol 2013;15:607-17.
15. Garzon MC, Huang JT, Enjolras O, Frieden IJ. Vascular malformations: Part I. J Am Acad Dermatol 2007;56:353-70.

16. Lance EI, Lanier KE, Zabel TA, Comi AC. Stimulant use in Patients with Sturge-Weber Syndrome: Safety and Efficacy. Abstract Presented at Child Neurology Society; 2012.

Author Queries???

AQ2:Kindly check author name doesn't match with reference list 